\title{
Development of a Low-cost Pressure Sensor
}

\author{
L. RÓNAI ${ }^{1}$, J. LÉNÁRT ${ }^{2}$, T. SZABÓ3 \\ 1University of Miskolc, Faculty of Mechanical Engineering and Informatics, Robert Bosch Department of \\ Mechatronics, ronai.laszlo@uni-miskolc.hu \\ 2 University of Miskolc, Faculty of Mechanical Engineering and Informatics, Robert Bosch Department of \\ Mechatronics, lenart.jozsef@uni-miskolc.hu \\ ${ }^{3}$ University of Miskolc, Faculty of Mechanical Engineering and Informatics, Robert Bosch Department of \\ Mechatronics, szabo.tamas@uni-miskolc.hu
}

Abstract. In this paper development of a pressure sensor has been discussed. The main benefit of this sensor is relatively cheap compared to commercially available ones. The unit contains a beam type load cell and a pneumatic cylinder. The load cell has 4 strain gauges in Wheatstone bridge configuration in order to convert the change of resistance into measurable voltage. The system is well applicable in numerous circumstances, where digital pressure signal is necessary for control or data acquisition.

Keywords: load-cell, microcontroller, pressure sensor

\section{Introduction}

The history of pressure measurement looks back to the old times. In the 17th century the first barometer, which could evaluate the atmospheric pressure, was invited by the Italian physicist Evangelista Torricelli. Blaise Pascal used the barometer to prove that the atmospheric pressure at the summit of a mountain is smaller than the flatland [1]. The Bourdon Tube was patented by Eugene Bourdon in the 19th century. The unit is applicable to perform relative pressure measurements. By reason of the apace development of the digital technology in the last century, several types of pressure sensors were appeared on the market.

Nowadays in engineering practice is essential to measure pressure, e.g., in robotics applications [2]. There are numerous electronic pressure sensors and transmitters available on the market [3]. Pressure sensors, transmitters are essential for pneumatic or hydraulic applications in the industry [4].

There are four commonly used pressure sensor types [5]: absolute, gauge, differential and sealed. An absolute pressure sensor measures the pressure relative to the perfect vacuum. A gauge pressure sensor provides the pressure relative to the atmospheric pressure. A differential pressure sensor measures the pressure difference of two independent measuring place. Sealed pressure sensor can measure the pressure and relates it to a fixed pressure value, which can be differ from the atmospheric pressure.

There are different pressure sensing principles, e.g., piezoresistive, capacitive, piezoelectric, and optical [2], [4], [5]. The piezoresistive principle utilizes the change of resistance of the strain gauges. These sensors are capable to measure quite small changes of the pressure. The capacitive sensors have a 
metallic diaphragm and a cavity chamber in order to set up a variable capacitor [4]. For highly dynamic changes in pressure the piezoelectric sensors are used [4]. This type of sensors utilizes the piezoelectric effect. Optical pressure sensors can detect the strain of an optical fibre [5].

The main purpose of this paper to develop a low-cost gauge type pressure sensor, which is capable to measure the pressure of such a pneumatic system. The base of the sensor is a beam type load cell, which has for strain gauges in Wheatstone bridge configuration.

This paper is organized as follows: Section 1 describes the elements of the developed pressure measuring device and its block scheme. An ATmega328 [6] type microcontroller processes the values of the acting pressure. The microcontroller is found on an Arduino Nano prototyping platform [7]. Section 2 contains the calibration of the unit, its test bench, and a result of a comparative measurement. In the last section the concluding remarks and the future plans are discussed.

\section{Scheme of the system}

The block scheme of the pressure measurement system is shown in Figure 1. A Rexroth Octagon type double acting pneumatic cylinder is used to exert force onto the load cell. The pressure, which acts the piston of the cylinder in chamber +, is written as:

$$
p=\frac{F}{A},
$$

where $F$ denotes the force, which exerts on the surface of the load cell, and $A$ is the area of the cross section of the piston.

Four strain gauges is found on the surface of the load cell in a Wheatstone bridge configuration.

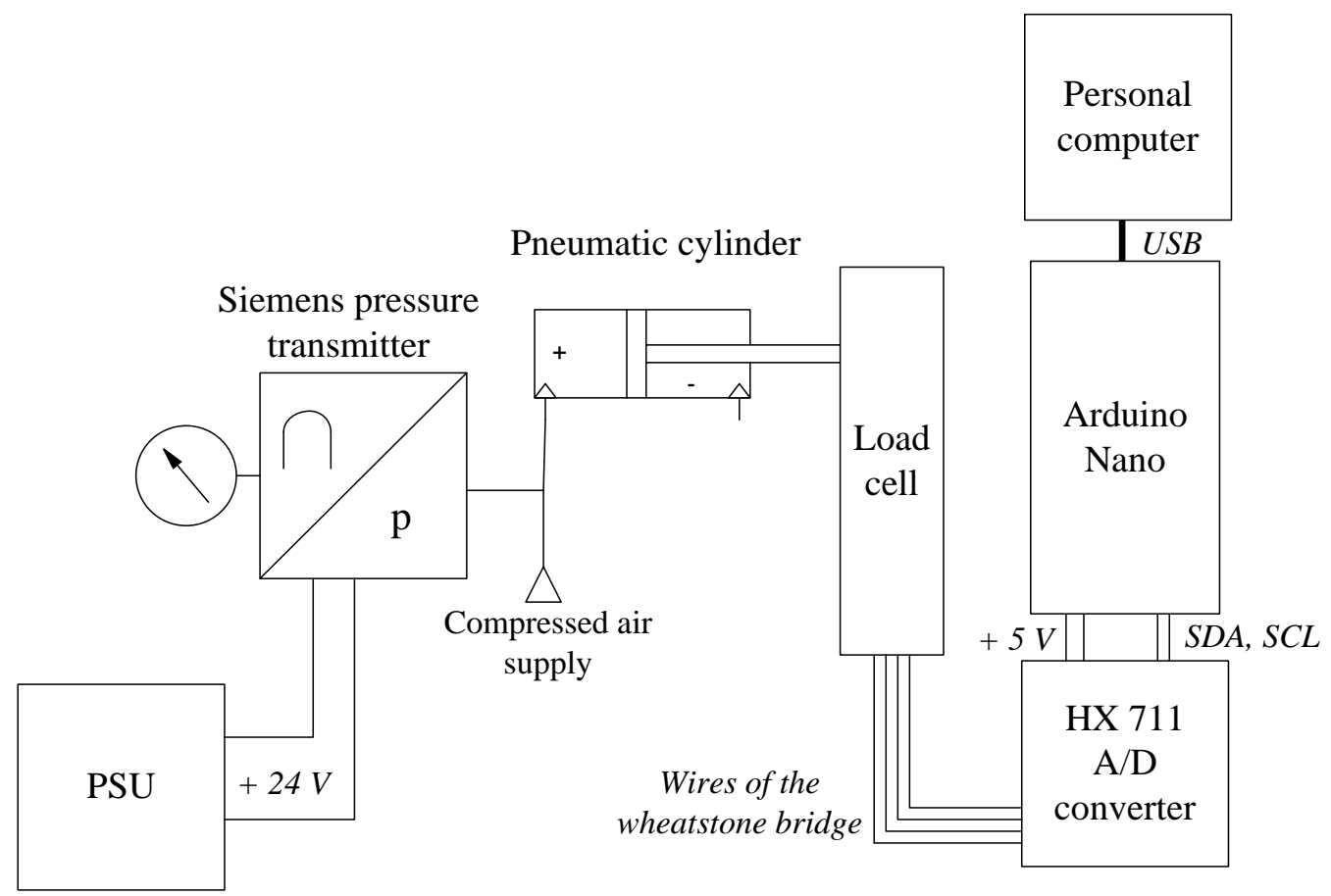

Figure 1. The block scheme of the system 
The bridge output voltage is measured and amplified by a HX711 24-bit sigma-delta analogue-digital (A/D) converter [8]. The unit has a serial data (SDA) and a serial clock (SCL) pins in order to build up the serial communication with Arduino Nano platform. The operating voltage of the microcontroller, and the A/D converter is $5 \mathrm{~V}$ TTL logic level. A personal computer can be attached to the microcontroller via Universal Asynchronous Receiver-Transmitter (UART) communication protocol in order to log and visualize the measured pressure values.

In the course of the calibration process of the system a Siemens pressure transmitter is connected to the chamber + of the pneumatic cylinder. The pressure transmitter has a digital screen where the actual pressure values can be monitored or it has a $4-20 \mathrm{~mA}$ output option, which can be processed by a data acquisition unit (DAQ). A 24 V laboratory Power Supply Unit (PSU) is used to ensure the recommended voltage for the pressure transmitter.

The calibration process of the load cell was performed in different pressure levels to get a coefficient, which ensures that the microcontroller can measure the pressure in bar metric unit.

\section{The test bench of the unit}

A test bench for the pressure measurement system is set up. Holder plates of the pneumatic cylinder, and the load cell were designed and manufactured. The technical drawing of the holder plate of the load cell is shown in Figure 2.
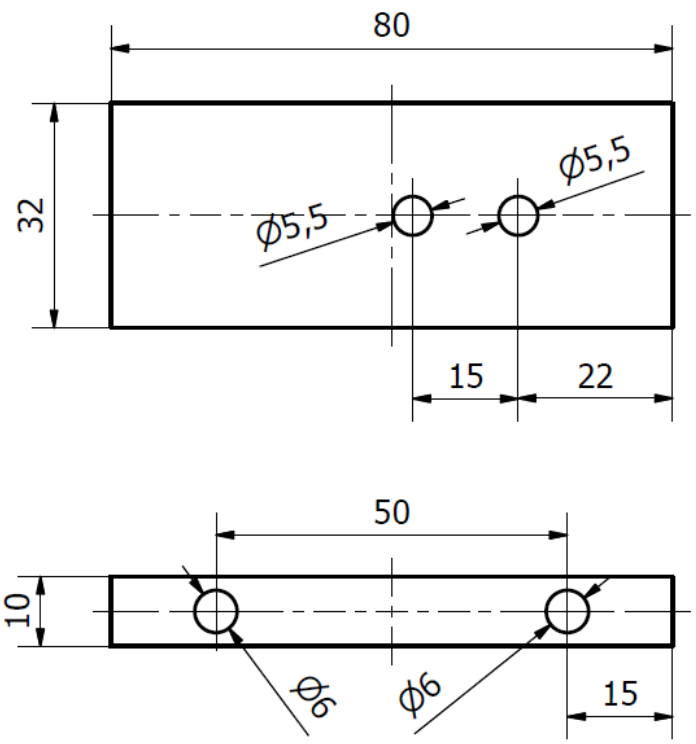

Figure 2. Holder plate of the load cell

The test bench of the system is shown in Figure 3. An aluminium T-slot plate serves the basis of the units. The pneumatic cylinder, and the load cell are mounted onto the T-slot plate via the holder elements. The electrical wiring is performed with the help of a breadboard, which makes the wiring process slightly easy (see Figure 4).

The pneumatic piping was performed via $4 \mathrm{~mm}$ inner diameter pneumatic tubes. The test system contains a compressed air can, which produces the compressed air. 


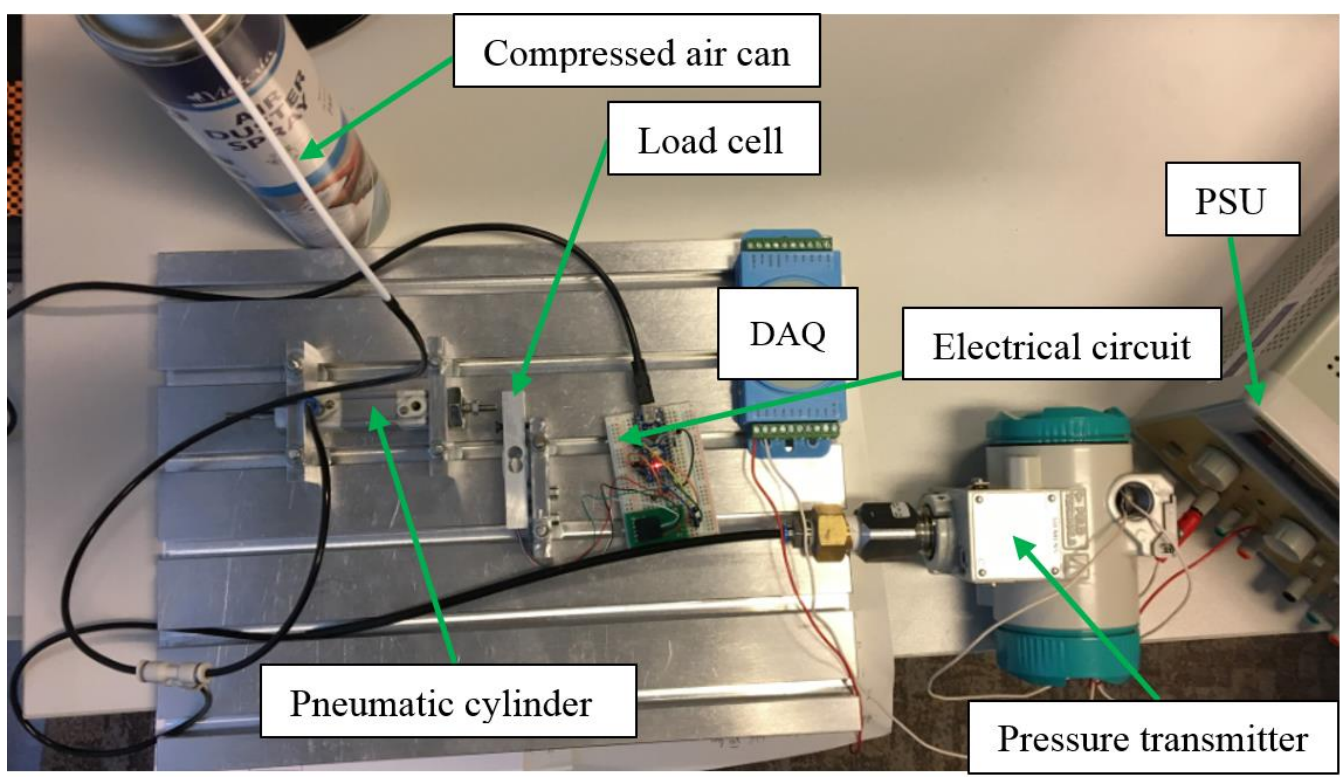

Figure 3. Test bench of a test measurement

The C program code of the system was written in Arduino IDE environment. The void loop 0 part of the program is shown in Table 1. This section of the program same as the while (1) statement.

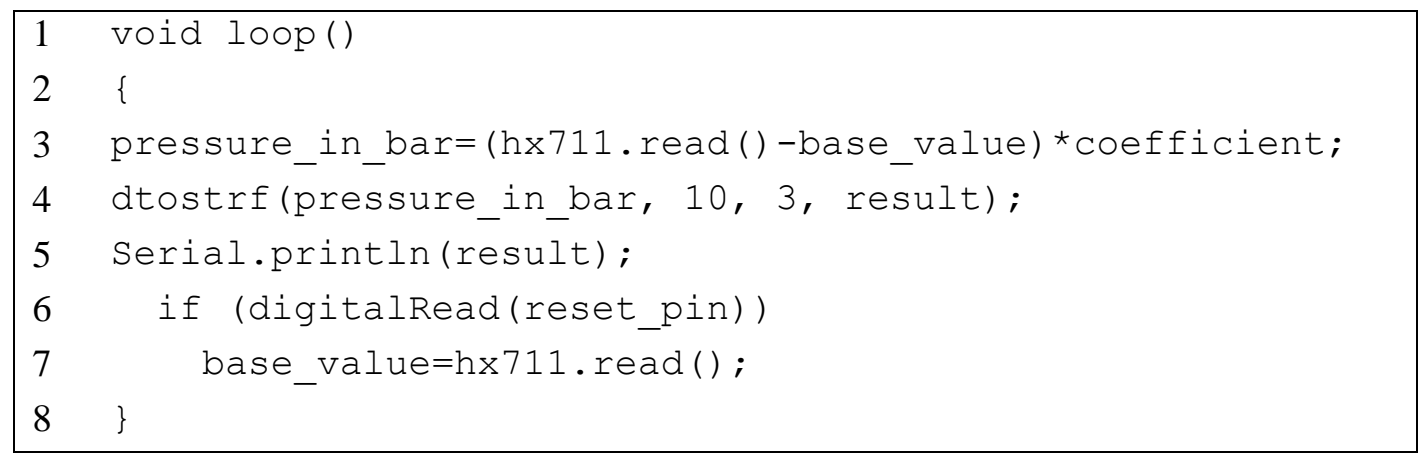

Table 1. The loop section of the program

The first row represents the beginning of the infinite loop. In the third row the program computes the actual pressure value. The $h x 711$.read $($ function produces the actual raw value of the A/D converter. The variable base_value represents the unloaded value of the load cell. The coefficient is a constant, which was determined in the course of the calibration process. The fourth row contains a type conversation, which converts the float type variable (pressure_in_bar) into a string type variable (result). The function has four parameters: float variable, string length, number of digits after the decimal point, and the result string.

In the next row, the Serial.println () function provides the computed and converted data to be sent to the computer in ASCII text followed by carriage return and newline characters.

The electrical circuit contains a micro switch (see Figure 4) in order to set the base value of the load cell. Rows 6, 7 contain an if statement, which provides the determination process of the base_value variable. 


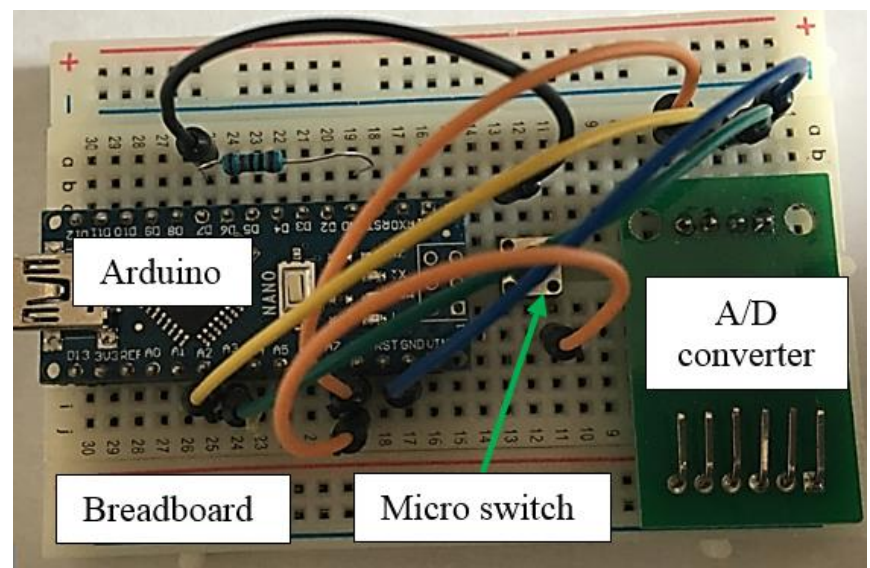

Figure 4. The electrical circuit

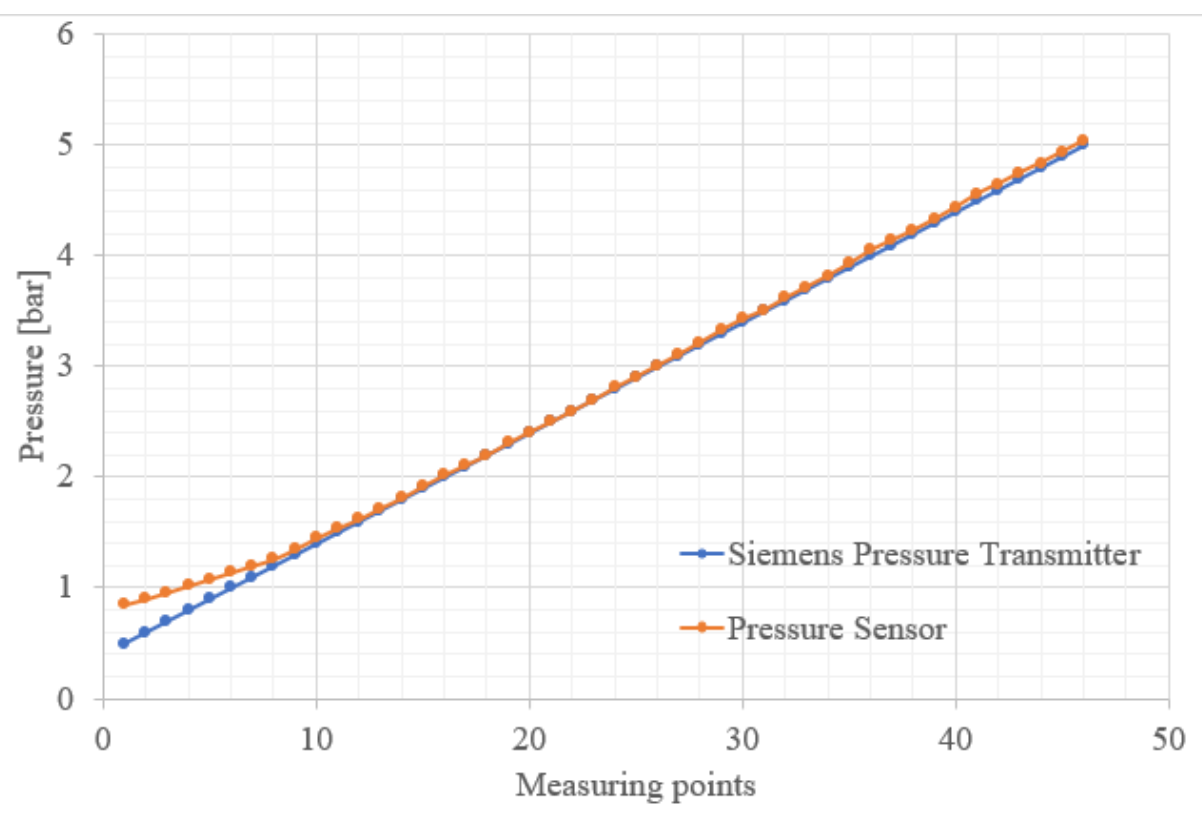

Figure 5. Comparison of the designed pressure sensor to the Siemens transmitter

In order to check the accuracy of the developed pressure measuring device in different pressure levels, a laboratory comparative measurement was also performed in 0.1 bar steps in a pressure range of $0.5-$ 5 bar. The obtained values are displayed in Figure 5. It can be seen that the measured values show good agreement in the range of 1.5-5 bar. If the pressure is below 1.5 bar the friction in the pneumatic cylinder cause significant error in the measured values.

Therefore, the designed pressure is well applicable above 1.5 bar.

\section{Conclusions}

The development of a low-cost pressure sensor is investigated in this paper. The system is capable to use with pneumatic circuits, where pressure measurement is essential. A test bench was designed to perform the calibration of the load cell and make test measurements. An Arduino prototyping platform and a 24-bit sigma-delta analogue digital converter were used to convert and transmit the data of the bridge voltage of the strain gauges to a personal computer via UART communication protocol. 
In the course of the calibration process, a Siemens brand pressure transmitter has been used to check the accurate pressure value. The coefficient, which is in the $\mathrm{C}$ program code, was determined in order to get the pressure results in bar metric unit. The developed pressure sensor has sufficient accuracy between 1.5 - 6 bar in laboratory conditions.

In the future the test bench will be attached to a pneumatic system to measure pressure values, e.g., pressure of chambers of a pneumatic cylinder. Furthermore, the size of the system will be reduced in order to get a compact size.

\section{Acknowledgement}

The described article was carried out as part of the EFOP-3.6.1-16-2016-00011 "Younger and Renewing University - Innovative Knowledge City - institutional development of the University of Miskolc aiming at intelligent specialisation" project implemented in the framework of the Szechenyi 2020 program. The realization of this project is supported by the European Union, co-financed by the European Social Fund.

\section{References}

[1] Sauvage, P., de Hosson, C. (2010) 'Approching the concept of atmospheric pressure: an interview based on Torricelli's barometer', GIREP, Reims, France., pp. 1-6.

[2] Almassri, A. M., Wan Hasan, W. Z., Ahmad, S. A., Ishak, A. J., Ghazali, A. M., Talib, D. N., Wada, C. (2015) 'Pressure Sensor: State of the Art, Design, and Application for Robotic Hand', Journal of Sensors, Hindawi Publ. Corp., pp. 1-12.

[3] TE connectivity (2016) 'Pressure Sensors', Datasheet, SS-TS-TE100, pp. 58-67.

[4] Graßmann, E., Gries, A. (2010) 'Electronic Pressure Measurement $\neg$ Basics, applications and instrument selection $\neg$ ', Süddeutscher Verlag onpact GmbH, 81677 Munich.

[5] Sikarwar, S., Singh, S., Yadav B. C. (2017) 'Review on Pressure Sensors for Structural Health Monitoring, Photonic Sensors', 7(4), pp. 294-304.

[6] Atmel (2009) '8-bit AVR Microcontroller with 4/8/16/32K Bytes In-System Programmable Flash', Datasheet, Rev. 8025I-AVR-02/09.

[7] Arduino prototyping platform: https://www.arduino.cc/

[8] AVIA Semiconductor (2016) '24-Bit Analog-to-Digital Converter (ADC) for Weigh Scales', Datasheet. 